\title{
Personnels et services de surveillance de la préfecture de police : de la constitution des dossiers de surveillance à la mise en forme du politique (1870-1900)
}

\section{Nathalie Bayon}

\section{OpenEdition}

Journals

Édition électronique

URL : http://journals.openedition.org/conflits/997

DOI : $10.4000 /$ conflits.997

ISSN : $1777-5345$

Éditeur :

CCLS - Centre d'études sur les conflits lilberté et sécurité, L'Harmattan

Édition imprimée

Date de publication : 1 mars 2004

Pagination : 83-98

ISBN : 2-7475-6791-5

ISSN : 1157-996X

\section{Référence électronique}

Nathalie Bayon, «Personnels et services de surveillance de la préfecture de police : de la constitution des dossiers de surveillance à la mise en forme du politique (1870-1900) », Cultures \& Conflits [En ligne], 53 | printemps 2004, mis en ligne le 04 octobre 2004, consulté le 30 mars 2021. URL : http:// journals.openedition.org/conflits/997 ; DOI : https://doi.org/10.4000/conflits.997

Ce document a été généré automatiquement le 30 mars 2021

Creative Commons License 


\title{
Personnels et services de surveillance de la préfecture de police : de la constitution des dossiers de surveillance à la mise en forme du politique (1870-1900)
}

\author{
Nathalie Bayon
}

La question de la surveillance politique des opposants, des groupes et des leaders contestataires est une préoccupation constante des différents régimes politiques qui se succèdent au XIXème siècle. La transformation des pouvoirs de l'Etat, et la diffusion des pratiques démocratiques (comme le suffrage universel le 5 mars 1848) conduisent les gouvernements successifs à modifier les pratiques de surveillance pourtant anciennes ${ }^{1}$. Il convient de maintenir leur efficacité, tout en les rendant plus discrètes afin qu'elles ne paraissent pas attentatoires aux libertés publiques. Des réformes s'opèrent sans jamais remettre en cause l'une des principales fonctions de la police qui est de garantir la sûreté de l'Etat, et donc de protéger le régime.

Le Second Empire réalise en dix ans une transformation radicale des moyens et des techniques, et met en place une puissante structure de contrôle de l'opinion et des formes d'expression qui constituent le pilier du régime ${ }^{2}$. Le pays dans son ensemble se pense sous la surveillance de la police, qui, par ses rapports, la peur et la répression, interdit l'existence de toute opposition - dans la logique de Fouché, pour qui « gouverner c'est faire croire ». Cette situation est progressivement dénoncée par le parti républicain qui se restructure dans le courant des années 1850 après la répression dont il fut victime au sortir du coup d'Etat ${ }^{3}$.

Dès 1869 , en dépit de l'apparente stabilité de l'Empire, les élus républicains réclament, au nom de la démocratie, la cessation immédiate de l'utilisation des forces de police à des fins politiques. 
L'Empire s'effondre le 2 septembre 1870 à Sedan et le 4 septembre, la République est proclamée. Toutefois, malgré cet avènement, on ne peut considérer les années qui séparent cet événement de l'élection de Jules Grévy à la présidence de la République (30 janvier 1879), comme une période "républicaine » tant ses partisans étaient exclus du pouvoir ${ }^{4}$.

Parvenus enfin au gouvernement, les républicains hésitèrent, ne purent ou ne voulurent se priver de ce pouvoir politique ${ }^{5}$ et s'ils réformèrent la partie visible de cet iceberg, ce fut afin de mieux le conserver, le modifier et même le développer.

Toutefois, un régime "démocratique " ne pouvait se concilier avec la définition de "police d'attaque» mise en oeuvre par Napoléon III et pensée par Fouché. Aussi, progressivement, glisse-t-on vers une police dite« de pure observation ». Néanmoins, les discours en la matière diffèrent considérablement des pratiques et les républicains eurent à leur tour recours à la manipulation politique, à la surveillance des hommes de gouvernement sous prétexte de les protéger, à l'emploi, volontaire ou non, d'agents agitateurs, et à des méthodes portant atteinte aux droits élémentaires de la vie privée, ainsi qu'à la mise en fiche d'une partie de la population. Ces procédés, et ce point est central, furent décriés par l'opposition républicaine au nom de la démocratie, et conservés par les républicains de gouvernement au nom de la sauvegarde du régime démocratique...

A cette période, les changements qui marquent la police de surveillance sont davantage des réformes cosmétiques que des modifications profondes. En effet, le régime républicain à la différence du précédent, reconnaît les oppositions - même s'il oeuvre de manière à exclure du jeu politique légitime les agents porteurs de discours plus radicaux - et ces dernières, agissant comme autant de repoussoirs, l'obligent - ou du moins tentent de l'obliger - à mettre le plus possible en adéquation ses discours et sa pratique. Aussi, c'est la nécessité de défendre la jeune République qui est sans nul doute le facteur qui conduit les républicains à maintenir les services de police politique.

Dans ce processus, le préfet de police occupe une place centrale car il est à la fois l'émissaire du gouvernement dans la police et l'ambassadeur de la Préfecture de police auprès du gouvernement. Homme politique lui-même à cette période, il use des dossiers de la surveillance dans le jeu politique, davantage d'ailleurs par leur détention que par une utilisation réelle.

En effet, ces dossiers dont nous nous proposons de restituer empiriquement les logiques de constitution, ne contiennent le plus souvent que des informations de faible valeur stratégique, quand elles ne sont pas simplement erronées ou inventées. Ils nous renseignent par contre sur les agents sociaux qui les constituent. Le constat de la double permanence des personnels et des pratiques au travers des bouleversements politiques liés à la transition à la démocratie ${ }^{6}$ nous invite à nous interroger sur la place de ces agents dans la définition du nouveau régime. En effet, incarnant dans cette période de fluidité politique ${ }^{7}$ un pôle de stabilité sur lequel les gouvernants successifs vont s'appuyer, ces agents vont contribuer, par les mises en forme qu'ils opèrent, à la délimitation de l'espace politique légitime.

Le préfet entre politique et administration

Le préfet de police de Paris dispose depuis l'arrêté du 12 Messidor an VIII de la République (1800) d'un pouvoir - policier, judiciaire, politique - colossal, contrebalancé par son placement sous l'autorité directe du ministre de l'Intérieur. Or, l'instabilité politique des débuts de la Troisième République délite ces liens. Cette émancipation du 
préfet est encore avantagée par le cumul autorisé de la fonction avec le mandat de député. Les premiers préfets de la République des républicains se pensent d'abord comme des hommes politiques. Andrieux disait à ce sujet: «C'est un député bien plus qu'un fonctionnaire qu'on appela en ma personne à la préfecture de police $»^{8}$.

Ce double positionnement permet au préfet d'intervenir dans le débat politique en tant que député pour défendre les positions prises par lui, mais également d'utiliser les services de la préfecture de police pour défendre ses intérêts de député. En effet, la puissance que lui confère la surveillance politique - attribut légal de sa fonction - est un atout majeur dans le champ politique. Espionnant le simple citoyen comme le président du Conseil, on devine le pouvoir occulte qu'il tire - ou peut tirer - des informations ainsi recueillies.

Tout d'abord, il est le seul maître des renseignements dont il dispose, aussi peut-il influer sur le gouvernement en sélectionnant le type d'information qu'il fait remonter jusqu'à lui. En fonction d'intérêts propres et/ou de la protection de son office, il communique aux gouvernants des faits de nature à les inquiéter ou à les rassurer.

De plus, le préfet, par les rapports sur l'état d'esprit des populations communiqués au gouvernement, le persuade - pour partie - de l'impérieuse nécessité de ne pas priver le nouveau régime de la protection que lui assure la préfecture de police.

Dans un système double, la fonction de préfet de police permet donc à celui qui l'occupe de concentrer entre ses mains un pouvoir politique important et d'être en capacité de l'utiliser, soit contre le gouvernement, soit de s'en servir à des fins personnelles. Les préfets usent donc de leurs prérogatives pour participer au jeu politique.

Toutefois, contrepartie de leur pouvoir politique, l'instabilité des préfets nuance fortement le pouvoir que leur confère leur statut. Du 4 septembre 1870 à 1889, treize individus occupent ce poste, exception faite des trois membres de la Commune. Il faut attendre 1893 et la nomination du préfet Louis Lépine pour voir s'inverser la tendance.

Mais l'instabilité qui touche la fonction de préfet ne concerne pas l'ensemble du personnel qui se caractérise, au contraire, par sa permanence. En effet, l'étude de l'état du personnel par grade et mention de la date d'entrée à la préfecture de police est édifiante, car elle révèle que $92 \%$ des employés gradés sont entrés en fonction avant 1870 , sans que cela ne porte atteinte au déroulement des carrières individuelles.

Si on considère la date d'entrée dans l'administration des deux principaux chefs de la police municipale - Caubet et Fontaine - il apparaît qu'ils sont respectivement recrutés en 1870 et $1854^{\circ}$. Il en est de même pour les deux inspecteurs divisionnaires entrés en fonction respectivement en 1846 et 1855 . Toutefois, cette constatation n'est pas propre au sommet de la hiérarchie et peut être généralisée à la quasi totalité des services de la préfecture de police ${ }^{10}$.

On trouve les mêmes mesures pour les services spéciaux dont, par exemple, ceux qui sont affectés à la surveillance des halles et des marchés ${ }^{11}$.

Ce point est central car c'est la collecte de renseignements qui constitue l'occupation principale de la police politique qui assure le pouvoir du préfet et également de l'institution. Les agents - policiers et informateurs - occupent une place prépondérante dans ce processus puisque c'est de leur travail et de ces logiques que dépend en dernière analyse la constitution des dossiers qui peuvent être utilisés dans le jeu politique. 
Qu'est-ce qu'un dossier de surveillance?

Dans sa définition la plus simple, le dossier est une sorte de biographie approximative d'une personne vivante, rédigée à son insu par la police. Au regard des principes républicains, il viole la loi sur la protection de la vie privée, dans ses espaces juridiquement définis.

La chronologie de la surveillance est très aléatoire et répond à des intérêts spécifiques, sans se soucier de la chronologie biologique des individus concernés. Etre à un moment donné l'objet d'une surveillance n'a pas un caractère irréversible - même si pour les hommes politiques c'est généralement le cas. L'objectif premier de ce procédé n'est pas à proprement parler de réunir des informations permettant d'établir la vérité, mais seulement de rapporter des faits, des paroles, des éléments divers, concernant un individu donné : «Le dossier n'a pas seulement pour but de faire connaître qui vous êtes, mais surtout ce qu'on a dit de vous $»^{12}$.

La surveillance n'est donc en rien un geste anodin, car du simple fait de son existence, elle transforme la condition du surveillé et le fait passer du statut de citoyen à celui de coupable potentiel. Le personnage surveillé est vu comme quelqu'un de dangereux, ce qui en réalité ne procède au départ de rien de tangible. Aussi, les agents ne sachant pas réellement ce qu'ils cherchent, construisent chaque fait relaté comme un indice. La police n'est donc pas à la recherche de la vérité mais d'un coupable : L'agent sert moins à surveiller les gens qu'à préparer la calomnie $»^{13}$ disait Yves Guyot en 1884. Ce faisant, la surveillance participe d'une vision policière du monde car ses raisons latentes sont d'épuiser, contrôler voire de combattre ceux qui ne violent pas vraiment la loi mais ont des opinions politiques et/ou mènent une vie qui va à l'encontre des valeurs dominantes de la société.

Le choix qui préside à la surveillance d'un individu ou d'un groupe ne résulte pas de principes rationnels et/ou préalablement définis, même si des critères comme l'activité politique ou artistique sont le plus souvent déterminants. Toutefois, ces catégories ne sont pas les seules à faire l'objet de la surveillance policière. Louis Andrieux écrit dans ses mémoires : Tout Paris a son dossier ». Néanmoins il relativise aussitôt son propos en précisant que «les dossiers bleus sont(...), les plus nombreux et les plus variés. Ce sont les dossiers politiques ou mondains $»^{14}$.

Malgré des cibles relativement standardisées, les critères de l'activité de surveillance varient abondamment en fonction de la préoccupation et des questions perçues comme dangereuses pour la sécurité de l'Etat - à un moment donné. Aussi peut-on tirer l'impression d'une augmentation des activités contestataires, quand il y a eu seulement déplacement des limites des comportements considérés comme légitimes ${ }^{15}$.

La surveillance varie également en relation avec les moyens matériels que la police détient pour y répondre. En effet, comme l'écrit Gary T. Marx, "La définition que se donne une société de la maladie mentale dépend en partie des facilités dont elle dispose pour y remédier $»^{16}$.

A l'époque étudiée ici, la majeure partie des dossiers concerne les hommes politiques élus ou non - toutes tendances confondues. On ne peut pas clairement établir, qui, des conservateurs ou des républicains, fait l'objet d'une plus grande surveillance. Il semble que celle-ci soit également répartie, ce qui contraste avec le discours des républicains de gouvernement qui concevaient la surveillance comme un "mal nécessaire " pour assurer la défense et la protection de l'Etat démocratique. Cette définition aurait dû en exclure de fait la surveillance des représentants de l'Etat. Mais leurs options politiques 
de la surveillance déclenchent des routines qui leur préexistent, comme le montre la surveillance des ministres républicains à cette période. Si la réactivation des pratiques de surveillance politique est antérieure à la République des républicains, il n'en demeure pas moins vrai que l'on observe dans ce domaine une continuité surprenante, tant dans l'intensité que dans les routines du travail de surveillance. Toutefois, une analyse des listes et des dossiers nominatifs révèle à quel point cette attitude et - ce qui est davantage significatif - ses résultats échappent dans une large mesure aux hommes de gouvernement.

Nous l'avons dit, la police de renseignement n'agit pas en fonction de critères précis. Elle exerce ses prérogatives à tous les niveaux de l'échelle sociale, surveillant indifféremment le simple citoyen - devenu suspect - et l'homme politique le plus en vue.

Les logiques de constitution des dossiers

Dans les années qui nous occupent, les dossiers ne procèdent pas d'une mise en forme standardisée. Les informations, communiquées à la préfecture de police par les agents de police, les informateurs, voire de simples citoyens, sont rassemblées pêle-mêle sans être vérifiées, recoupées ni même hiérarchisées. A la lecture, il semble que le but soit de réunir autant d'informations que possible, sans prendre en compte leur niveau d'intérêt. Aussi, les dossiers contiennent-ils beaucoup de détails mais également beaucoup de redites et d'erreurs plus ou moins grossières.

Le service chargé de fabriquer matériellement le dossier ne semble pas procéder selon une méthode précise, même si au final tous les dossiers ont une apparence semblable. Ils regroupent des informations le plus souvent d'ordre général, quelques détails de la vie privée, voire intime.

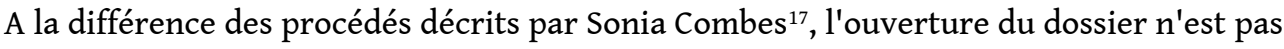
précédée par la confection d'une petite biographie, même sommaire, de la personne surveillée. Seuls figurent son nom et le numéro de classement qui lui a été attribué. Le lecteur est donc directement confronté à un papier relatant un fait dont il ignore le plus souvent la nature. Toutefois, les formes de documents recueillis ne sont pas très variées.

Seulement trois types de pièces constituent la part la plus importante du dossier : les coupures de presse, les rapports rédigés par les agents de police et ceux des informateurs. A ces rapports, qui sont le fruit d'une surveillance indirecte concernant le plus souvent la vie publique d'un individu, s'ajoutent des télégrammes et des copies de courrier personnel - en petit nombre il est vrai - attestant de l'intrusion policière épisodique dans leur vie privée. Ensuite sont regroupés - sans que l'on puisse dégager de logique - des documents qui semblent davantage avoir été glanés par hasard qu'être le fruit d'une recherche précise. On trouve ainsi des photographies, des caricatures, des affiches électorales, des cartes de visite, des menus de banquets, des invitations. Ce corpus est complété par des indications extraites de dictionnaires biographiques genre alors très en vogue - ainsi que de quelques brochures écrites par le surveillé.

Ainsi, peu à peu, à la lecture des documents, se dessine une représentation partielle et orientée de la vie d'un individu. Par ce travail, la police ne cherche pas à établir une quelconque vérité, mais à réunir des éléments concernant un personnage et au-delà, la manière dont il est perçu par ses contemporains : "Aussi mettra-t-on dans votre dossier pêle-mêle sans distinguer entre le vrai et le faux tout rapport dont vous aurez été l'objet, toute dénonciation vous concernant, tout article de journal, tout fait divers où vous serez nommé $»^{18}$. 
Toutefois, la police doit se satisfaire de ce type de renseignements car elle ne paraît pas en capacité d'obtenir ni des informations plus originales, personnelles, ni même de vérifier la plupart de celles qu'elle recueille. En effet, devenues archives, les erreurs contenues dans les dossiers choquent l'oeil de l'historien qui les consulte.

Il semble que les différentes informations qui remontent jusqu'au préfet soient simplement lues - et encore, considérant la masse de renseignements collectés quotidiennement, il est peu probable que toutes le furent - et ensuite classées en l'état.

Il est intéressant de remarquer que le dossier ne permet jamais de reconstituer une fiche biographique d'un individu, même la plus élémentaire. La préfecture de police ne prend pas la peine de manière systématique de se procurer des données administratives officielles, comme des actes de naissance, des actes notariés, etc. Les renseignements de ce type sont présents par le biais d'informations récoltées dans les dictionnaires et/ou des articles de journaux. Chaque scrutin voit fleurir dans la presse des séries de biographies, dont la fiabilité n'est pas la caractéristique première. Tous les dossiers contiennent plusieurs dizaines de coupures de journaux de ce style, dont la présence nous informe davantage sur les pratiques du personnel chargé de dépouiller la presse que sur l'individu surveillé lui-même.

En effet, alors que le nombre des publications parisiennes et nationales est très important, on retrouve presque toujours les mêmes titres dans les cartons de la préfecture de police ${ }^{19}$ : des journaux conservateurs (Le Pays, L'univers, Le Figaro); des journaux républicains modérés (Le Temps, La République française, Le Siècle); des feuilles radicales (Le Rappel, La Lanterne, L'Evénement, Le cri du peuple). Chaque journal est lu, découpé, puis classé dans le dossier correspondant, sans annotation ni ordre. Aussi, voit-on défiler une masse considérable d'articles se paraphrasant les uns les autres, ayant été sélectionnés parce qu'ils parlent d'un individu soumis à la surveillance de la police de renseignement. La méthode employée pour dépouiller la presse correspond dans une large mesure au mode par lequel on recueille l'information dans la rue et dans les espaces publics : on rapporte toutes les rumeurs, les ragots, les « on dit », etc.

On aboutit à des conclusions semblables en ce qui concerne les rapports rédigés par les agents de police. Même s'ils n'ont pas d'objet prédéterminé, ils relatent le plus souvent des réunions publiques. On peut aisément percevoir l'intérêt pour le préfet de savoir ce qui est dit du haut des tribunes ainsi que l'ambiance de la réunion, le nombre de participants, la durée, etc. Cependant, on constate que les rapports émanent de divers services - qui n'ont pas de liens entre eux - et sont fréquemment chargés de surveiller les mêmes rassemblements. Leur lecture est répétitive car ils relatent les mêmes faits sur les mêmes modes.

Une telle analogie montre que les agents de la préfecture de police ignorent réciproquement la présence des autres. Cette double surveillance semble davantage être le résultat du hasard que d'une volonté de la préfecture de police de s'assurer un double regard. Surtout que dans ce genre de réunion, outre l'ambiance, le résumé des discours et quelques ragots, que peuvent bien apprendre de capital les agents présents? D'autant que les gens surveillés sont conscients de l'être. Un nombre très important de leurs témoignages en atteste, cela transparaît même dans les rapports des agents : "Castel se flattait aux mesures qu'il avait prises, d'empêcher la préfecture de police d'envoyer au banquet un seul de ses mouchards $»^{20}$.

Les surveillés ne sont pas dupes de la surveillance dont ils sont l'objet, ce qui accroît la difficulté du travail des agents de renseignement, car non seulement leur cible se méfie 
mais elle peut aussi les instrumentaliser pour faire remonter de faux bruits jusqu'au préfet.

Les rapports sont donc peu précis, ils rendent compte soit du visuel, soit des bruits qui courent sur les boulevards, dans les lieux publics comme les cafés, etc. L'informateur officiel ou non - relate constamment ce qui se dit. C'est pourquoi la plupart des rapports contiennent des: "On dit que, on $m^{\prime} a$ dit que, il parait, etc. $»^{21}$. Leurs sources d'information sont des concierges, le petit personnel des journaux - par exemple - à quoi s'ajoutent leurs flâneries sur les boulevards, dans les bars qui leur permettent au grès des rencontres de discuter avec des passants, des connaissances, et de sonder ainsi «l'état d'esprit» de la population relatif aux individus surveillés. En effet, le plus souvent, l'agent n'est pas dans l'intimité de la personne qu'il surveille, il est donc dans l'impossibilité de lui soutirer directement des informations. Même les agents qui espionnent dans les cafés ne font pas partie du cercle qu'ils épient.

Il en est de même pour la surveillance des réunions tenues entre députés et/ou sénateurs. L'informateur n'est presque jamais à l'intérieur, aussi se contente-t-il de dire qui était présent et combien de temps a duré l'entrevue. De plus, il semble qu'il n'y ait pas un agent attaché à la surveillance d'un individu mais que toutes les brigades préposées à la surveillance fassent état au préfet de ce qui est porté à leur connaissance, sans être spécialement en charge de tel individu ou groupe.

Ces hommes ne prennent pas le temps de vérifier les informations qu'ils transmettent, ainsi le rapport suivant peut-il invalider partiellement ou entièrement le précédent, sans que cela ne semble avoir de conséquence pour le rédacteur. Cela se produit fréquemment avec les télégrammes annonçant le voyage et l'itinéraire d'un individu. Il sera sensé se rendre, par exemple, à Lyon, et le lendemain on signalera une autre destination, sans mentionner le motif de la confusion. Ou encore, l'agent informe le préfet des agissements d'une personne alors qu'il s'agit en réalité d'un membre de sa famille et non de lui-même ${ }^{22}$...

L'information n'est donc pas recueillie selon un protocole rationnel, ni même de façon à garantir sa véracité. Cette très faible fiabilité n'est pas inconnue des hommes qui président à leur constitution. A cet égard, faisons écho de l'expérience de deux préfets qui commentent la lecture de leur propre dossier.

Kératry consulte son dossier, constitué par l'administration de Napoléon III. A sa lecture « $i$ sourit de la nature et de la pauvreté des informations recueillies sur ses agissements politiques. Il écrivit la note suivante en marge de la première feuille : il est triste d'avoir dépensé autant d'argent pour obtenir si peu de renseignements et des renseignements si erronés ${ }^{23}$.

Citons également la réaction du préfet Andrieux à la lecture de son dossier personnel : "Je le garde au fond de ma bibliothèque avec toutes les calomnies grossières et les dénonciations haineuses qui sont d'ordinaire le fonds de ces sortes de documents. Et cependant tels qu'ils sont ces dossiers sont utiles, indispensables même pour les recherches de police, mais à la condition de n'être connus ni de particuliers, ni même de la justice et de n'être jamais ouverts que par des chefs de service expérimentés, sachant les lire et y démêler, parmi tant d'allégations inexactes et souvent contradictoires, la part de vérité qu'ils contiennent $»^{24}$.

Après une telle observation, on peut se demander pourquoi ils accordent autant de crédit à la nécessité de faire perdurer une pratique qui faisait si aisément état de ses limites. 
Il y a d'abord la volonté de cette administration - comme toute bureaucratie - de se perpétuer et de justifier son existence. Mais aussi une dimension plus structurelle de la surveillance politique. Posséder des dossiers - même vides ou faux - sur des individus qui savent et/ou pensent que le préfet les détient, lui confère un grand pouvoir. Il existe un véritable fétichisme de la surveillance politique que l'on retrouve, à bien des égards, jusqu'à nos jours ${ }^{25}$. Cette croyance en l'omniscience de la préfecture de police, qui se traduirait par une omnipotence, est partagée par nombre de responsables politiques, du gouvernement notamment. La surveillance politique devient dans ce contexte une technologie du pouvoir - au sens foucaldien du terme ${ }^{26}$ - par les formes d'autocensure et d'autocontrainte qu'elle génère, plus que par la répression qu'elle déchaîne. C'est cette dimension qui explique le maintien de ces pratiques, bien davantage que le souci de "savoir vrai».

Mise en forme et mise en ordre bureaucratique de l'Etat

Si le travail de surveillance trouve sa légitimité dans le fait même d'exister, la masse colossale d'informations collectées - jusqu'à 250 documents par jour - ne pouvait pas être exploitée telle quelle. Elle transite donc par des auxiliaires, les "notiers", occupant une place centrale dans leur mise en forme, qui est aussi une mise en ordre.

Chaque compte rendu fait l'objet d'un traitement de plus en plus sophistiqué et standardisé à mesure que l'on avance dans la période. Les rapports faits par les agents de police sont retranscrits dans un formulaire type, d'abord manuscrit puis imprimé. Ils résument d'une phrase le thème principal du rapport, et mentionnent ensuite son numéro de classement dans les archives. C'est ce document qui est présenté au préfet.

Le procédé est le même en ce qui concerne les rapports émanant des agents secrets qui sont recopiés dès leur arrivée par les «notiers ». Une fois la copie terminée, l'original est immédiatement détruit. Puis, sur le modèle des rapports des agents de police, ils sont résumés et transmis au préfet de police.

Ce travail de mise en forme répond à deux impératifs au moins.

Le premier est d'ordre bureaucratique : il faut pouvoir lire et utiliser des documents écrits par des acteurs dont le niveau de formation scolaire reste faible à cette période. De même, les informateurs, dont le profil social est proche de ceux qu'ils surveillent, sont dans leur grande majorité des hommes du peuple.

Le second renvoie davantage au maintien du caractère secret des personnels de la surveillance. Cette réécriture rend la traçabilité par la graphologie totalement impossible. Il reste donc très difficile de les identifier à moins de posséder le registre dans lequel figure l'identité de ces informateurs en face du numéro qui leur est attribué. Or ce registre n'est vraisemblablement ni à la préfecture de la police ni au CARAN. L'anonymat que garantissent ces procédures aux informateurs est particulièrement important pour les plus prestigieux d'entre eux et une condition nécessaire de la poursuite de leur collaboration.

L'étude de ces fiches standardisées montre des phrases correctes, où les fautes d'orthographe sont rares. Ceci nous renseigne sur le niveau scolaire au moins, et donc social, des agents de la brigade des notes. Cette équipe de lettrés est composée de trente-quatre inspecteurs qui dépendent du premier bureau ${ }^{27}$. Si les registres $d u$ personnel ne nous permettent pas d'en savoir davantage sur ces individus, ni sur la brigade des notes, on perçoit en creux leur importance dans la surveillance politique. 
En effet, par leur travail ordinaire de résumés, de corrections, ils formatent les informations collectées, quand ils ne les réinterprètent pas.

A ce travail s'ajoute le dépouillement de la presse, dans laquelle ils opèrent une sélection de ce qui est «important ». Si bien qu'en définitive le dossier de surveillance est la résultante du filtrage, tant en termes de volume qu'en termes de forme de l'information. Cette tâche n'est pas unique, elle est à mettre en parallèle avec d'autres fonctions de ce type qui se développent à cette époque, comme les greffiers du Parlement, qui en retranscrivant les discours des élus les réécrivent en leur donnant une forme conforme à l'institution. Or, cette sélection et cette mise en forme contraignent, bornent l'espace de décision de ceux qui en sont les destinataires. Ils peuvent se voir comme ce que Graham T. Allison appelle des «outputs organisationnels » ceux «qui structurent la situation à l'intérieur des contraintes étroites à partir desquelles les responsables doivent 'décider' à propos d'un problème. Les outputs posent le problème, fournissent les informations et font les premières démarches qui donnent une couleur à l'aspect du problèmes qui se présente aux responsables ${ }^{28}$.

Maitres des dossiers, ces fonctionnaires ont le monopole des termes dans lesquels vont se poser les choix. En discriminant les tenants d'une parole politique qu'ils jugent illégitime - jugement socialement constitué par leur double appartenance à la police et à la petite bourgeoisie des « clercs » - et en agissant pour les exclure du débat politique, ces fonctionnaires participent à la définition de la politique légale et à la pacification du jeu politique. En sériant de la sorte les frontières du politique, et en repoussant dans l'illégalité toutes les alternatives, ils contribuent à l'enracinement du régime républicain.

Mais plus encore, en inventant les routines et les procédures bureaucratiques de mise en forme de la surveillance, ils donnent un sens concret à l'idée de continuité de l'Etat au-delà de la philosophie politique qui le fondait.

C'est aussi de cette manière que peuvent s'appréhender les évolutions successives des discours des républicains de gouvernement, hier si prompts à dénoncer ces pratiques et qui considèrent désormais que le maintien de la police de renseignement est un mal indispensable pour protéger les nouvelles institutions.

En effet, ce travail administratif est loin d'être dépourvu de tout enjeu théorique, car il véhicule des représentations de l'Etat "qui sont constitutives de la réalité même de l'Etat parce[qu'ils]ont contribué durablement à sa formation et à sa transformation et [qu'ils] ont été d'autant plus efficaces[qu'ils]semblaient n'obéir qu'à des considérations pratiques $»^{29}$.

Ainsi, tout en assurant la pérennité de leurs fonctions et la permanence de leur institution, ce type particulier de personnels occupe une place importante dans la construction en actes de l'Etat républicain.

La permanence de leurs savoir-faire et des visions du monde qu'ils impliquent et supposent permet de porter un autre regard sur les «transitions démocratiques », bien au-delà des discours euphoriques qui les accompagnent. 


\section{NOTES}

1. . La police et les renseignements sont, selon Jean-Marc Berlière, des éléments indissociables qui caractérisent l'Etat moderne. La police moderne est née sous le règne de Louis XIV, "avec le renseignement et le nouvel usage qu'il impliquait de l'écriture du savoir et de l'archive ». Bélière J.M., « Police et renseignement », in Amiral Lacoste (dir.), Le renseignement à la française, Paris, Economica, 1998, pp. 9-27. Confère également L'Heuillet H., « Le renseignement ou l'impossible maîtrise du politique », Cahiers de la sécurité intérieure, n³0, Paris, 1998, pp. 103-118.

2. . Voir Bernard M., « La réorganisation de la police sous le Second Empire (1851-1858) : des bras infatigables ", in Vigier P. (dir.), Maintien de l'ordre et polices en France et en Europe au XIXème siècle, Paris, Créaphis, 1987, p. 119-135 ; Rigotard J.,La police parisienne de Napoléon, Paris, Tallandier, 1990, 498 pages.

3. . Au soir du 2 décembre 1851, les principaux chefs du parti républicain sont arrêtés ou contraints à l'exil. Voir Tchernoff I., Le parti républicain au coup d'Etat et sous le Second Empire, Paris, Ed. Pedone, 1906, 676 pages.

4. . Les républicains sont maîtres du pouvoir durant la période dite de Défense Nationale, (septembre 1870-février 1871). Puis les élections législatives portent à la Chambre une écrasante majorité de conservateurs dont l'objectif est de restaurer la monarchie parlementaire. C'est la République des ducs. Le parti républicain est à nouveau déstructuré à la fois par sa défaite électorale et par les événements de la Commune de Paris. Il lui fallut presque huit années pour parvenir à conquérir le pouvoir au prix de luttes constantes. Voir Halévy D., La fin des notables, La République des ducs, Paris, Grasset, 1937, p. 411 ou Mayeur J.M., Les débuts de la Troisième République, Paris, Seuil, 1973, p. 256.

5. . Machelon J.P., La République contre les libertés, Paris, FNSP, 1976, 462 pages.

6. Cette notion de transition démocratique, fortement remise en cause depuis les années 1990, fait l'objet de vives discussions. Pour notre part nous la mobilisons dans son sens premier et minimaliste, c'est-à-dire comme période floue de changement de régime où les idées républicaines cherchent à s'imposer.

7. . Sur la notion de fluidité des crises politiques, voir Dobry M., Sociologie des crises politiques, Paris, Presses de la FNSP, 1986, 319 pages.

8. . Andrieux L., cité par Jeanneney J.N., « Les provocations du préfet Andrieux », La guerre des polices, L'Histoire, février 2000, pp. 48-51.

9. . Il est vrai que sous l'Empire ils étaient cinq. Leur nombre a baissé à cause du non renouvellement à l'occasion des départs à la retraite.

10. . Dans les bureaux, sur dix-neuf employés, dix-sept sont recrutés avant 1870, un en 1872 et le dernier en 1878. La composition des brigades de sûreté est tout aussi révélatrice de cette stabilité. Les vingt-sept personnes constituant le corps des commissaires de police et des sous-brigadiers étaient toutes en poste avant 1871. C'est également le cas de la brigade des moeurs : sept individus gradés, tous intégrés avant 1871. Brigades des garnis : neuf individus gradés, huit recrutés avant 1870 et un en juin 1871. La proportion est quasiment identique dans les brigades de recherches : Première brigade de recherche : onze individus gradés, sept entrés avant 1871, et quatre après $(1871,1872,1873)$. Deuxième brigade de recherches : dix individus gradés, un seul recruté après 1871 . Troisième brigade de recherche : huit individus gradés, tous en 
poste sous l'Empire. Cinquième brigade de recherche : cinq individus gradés, tous également en poste avant le 4 septembre 1870. Sixième brigade de recherche : deux individus gradés, une recrue en 1865, l'autre en 1872. Voir Bayon N., « Transition démocratique et services de renseignements politiques : la surveillance des hommes et groupes politiques d'opposition à Paris au début de la Troisième république », Paris, rapport IHESI, décembre 2000, 73 pages.

11. Quatorze individus gradés, tous en poste sous l'Empire.

12. . Andrieux L., op. cit., p. 29.

13. . Guyot Y., La police, Paris, Charpentier, 1884, p. 161.

14. . Andrieux L., op. cit., p. 30.

15. . A cet égard le cas des anarchistes est particulièrement évocateur. Voir Maitron J., Le mouvement anarchiste en France, Tome 1 : Des origines à 1914, Paris, Maspero, 1975, p. 252.

16. . Marx G.T., «L'agent provocateur et l'indicateur », Sociologie du travail, $\mathrm{n}^{\circ} 3$, Paris, Seuil, 1973, (pp. 241-268), p.261.

17. . Combe S., Une société sous surveillance, les intellectuels et la Stasi, Paris, Albin Michel, 1999, p. 264.

18. . Andrieux L., op. cit., p. 29.

19. . Bien sûr cela varie en fonction de l'apparition et de la disparition très rapide des journaux à cette époque. Les titres cités ici sont ceux le plus fréquemment répertoriés. 20. . APP, Carton B/A 929, Rapport du service des garnis du 8.10.1876.

21. . Exemple de ce type d'approximation : « Gambetta serait parait-il invité cette année aux réceptions du corps diplomatique », 7 janvier 1879, APP B/A 916.

22. . Ceci est particulièrement fréquent dans le dossier d'Eugène Spuller, dont le frère Auguste Spuller est également investi en politique, toutefois celui-ci est nettement plus radical. Ce qui n'est pas sans entraîner des confusions que les agents de la préfecture de police ont bien du mal à démêler.

23. . Anonyme, 20 ans de police : souvenirs et anecdotes d'un ancien officier de paix, Paris, Ed. Dentu, 1881, pp. 142-143.

24. . Andrieux L., op. cit., p. 28.

25. . L'empressement des dirigeants socialistes parvenus au pouvoir en mai 1981, à consulter leurs dossiers des Renseignements généraux est un exemple significatif. 26. . Foucault M., Surveiller et punir, Paris, coll. Tel Gallimard, 1975, p. 360.

27. . Le 1er décembre 1884, une sous-commission de la préfecture de police est réunie pour démêler les contentieux avec le conseil municipal. A cette occasion le président Lyon-Alemand en présence du secrétaire général de la préfecture de police dit:

"Cependant le premier bureau et la première division sont bien politiques, et nous avons quelques intérêt à bien déterminer à qui doit en incomber la dépense ». In rapport de Pichon relatif au budget de la préfecture de police. P $44 \mathrm{du}$ n $^{\circ} 83$, Conseil municipal de Paris, 1884.

28. . Allison G. T., « Modèles conceptuels et la crise des missiles de Cuba », Braillard, P., Théories des relations internationales,PUF, Paris, 1977, p. 184. Plus largement, voir Allison, G.T., et Zélikow P., Essence of décision, New York, Longman, 1999, 416 pages.

29. . Bourdieu P., Christin O., et Will P.E., "Sur la science de l'Etat », Actes de la Recherche en Sciences Sociales, $\mathrm{n}^{\circ} 133$, Paris, juin 2000, p. 5. 
INDEX

Index géographique : France

Index chronologique : $19 \mathrm{e}$ siècle

Mots-clés : bases de données, histoire, police, Renseignement, surveillance 